\title{
4 Die Evolutionsgeschichte des Menschen
}

\begin{abstract}
Human evolution, at first, seems extraordinary. How could the process that gave rise to slugs and oak trees and fish produce a creature that can fly to the moon and invent the Internet and cross the ocean in boats? Was it some kind of divine spark that made our brains special? Well, I don't think so, because I think that you can understand human evolution in terms of the ordinary process of Darwinian natural selection.
\end{abstract}

Pinker (2001)

\subsection{Die Rolle der Paläoanthropologie in der Sprachursprungsforschung}

Ist die Evolutionstheorie der begrenzende sowie fixierende „Rahmen und Hintergrund" der Menschwerdung im Allgemeinen sowie der Sprachursprungsforschung im Speziellen, so bestimmt die Gesamtheit der paläoanthropologischen Erkenntnisse, also die Rekonstruktion der menschlichen Evolutionsgeschichte über einschlägige Fossilfunde, wenigstens theoretisch maßgeblich und kompromisslos das endgültige Bild der Menschwerdung und damit auch der Sprachursprungsforschung. Die Praxis zeigt sich entgegen dieser Feststellung als unübersichtlich und in vielerlei Hinsicht mehrdeutig, da sich die Fundlage bis heute als unzureichend und inhärent problematisch erweist. In diesem Sinne existiert eine große Zahl noch unbeantworteter Fragestellungen und viele der aktuell gemeinhin akzeptierten Interpretationen zu paläoanthropologischen Funden entsprechen möglicherweise nur näherungsweise - bzw. gegebenenfalls nicht - der Wahrheit. Diese in erster Linie sehr kritischen Aussagen sollten freilich nicht missgedeutet werden, denn die jahrzehntelange Arbeit einer Vielzahl von Experten zu bislang tausenden von Funden allein innerhalb der Gattung des Menschen bietet durchaus einen leistungsstarken Erkenntniskomplex, der grundsätzlich ein fruchtbares und aufklärendes Bild vorzeichnet - nur eben kein in allen Details ausgearbeitetes, abschließendes Bild.

Im nun direkt Folgenden sei in ähnlicher Weise wie bei der Umschreibung der evolutionsbiologischen Verwandtschaft einzelner Taxa als ein Stammbaum des Lebens im Kontext des vorigen Kapitels ein Einstieg in die Thematik durch die Dekonstruktion einer populären Metapher zum Forschungsfeld gegeben, bevor zu einer Skizze der Menschwerdung als dem maßgeblichen Inhalt des vorliegenden Abschnitts übergegangen wird. Auf diese Weise soll die tiefgreifende, inhärente Problematik der paläoanthropologischen Forschung, welche die trotz zahlreicher Fossilfunde noch immer ungenügende Forschungslage begründet, veranschaulicht werden. Die hierzu herangezogene Metapher ist 
durch das „Puzzle der Menschwerdung“ (vgl. bspw. die deutsche Ausgabe von Tattersall 1995, welche im Literaturverzeichnis als Tattersall 1997 angegeben ist) bzw. allgemeiner durch das „Puzzle (innerhalb) der Paläontologie“ gegeben und findet in populären Medien vielseitig Verwendung. Innerhalb eines solchen Vorgehens wird oftmals die außerordentliche Komplexität dieses paläontologischen bzw. paläoanthropologischen Puzzles betont, jedoch besteht innerhalb dieses Vergleichs eine unvermeidliche und umfängliche Ignoranz gegenüber den Schwierigkeiten, mit denen sich die Paläontologie generell - und im Speziellen die Paläoanthropologie - konfrontiert sieht.

\subsection{Zur Puzzle-Metapher in der Paläontologie}

Ein handelsübliches Puzzle liegt, sofern es nicht aus zweiter Hand oder in Form einer Fehlproduktion erworben wurde, in einer einheitlichen sowie konstruktiven Art und Weise vor. Mithin treffen die folgenden Eigenschaften dabei zu: Erstens ist die Größe bzw. die Teilchenzahl des Puzzles auf der Verpackung definiert. Zweitens ist das Puzzle vollständig. Drittens ist innerhalb der Verpackung jedes Puzzleteil nur ein einziges Mal enthalten. Viertens hat das Puzzle einen klar definierten Rand mit klar identifizierbaren Randstücken. Fünftens und letztens ergibt das Puzzle ein kohärentes, scharfes und zweidimensionales Bild. Mithin besteht das typische Vorgehen, ein derartiges Puzzle zu lösen, darin, alle Randstücke zu sammeln und in grobe Farb- bzw. Musterblöcke zu trennen. Mittels verwandt wirkender Randstücke und insbesondere von den Ecken ausgehend ist es in der Regel möglich, den Randbereich des Puzzles mit großer Effizienz auszulegen. Dieser begrenzende Rahmen ist während der darauf folgenden Schritte von großer Hilfe in dem Programm, das Puzzle als Gesamtheit sukzessive zu rekonstruieren.

Soll die Paläoanthropologie nun einem Puzzle gleichen, so reicht ein Hinweis auf „große Komplexität“ nicht aus, denn die einschlägige Forschung sieht sich vor Schwierigkeiten, welche bei einem handelsüblichen Puzzle unabhängig von dessen Komplexität nicht gegeben sind. Es ist jedoch möglich, die normative Situation eines gebräuchlichen Puzzles derart zu modifizieren, sodass einige der innerhalb der Paläoanthropologie ${ }^{19}$ bestehenden Probleme veranschaulicht werden können. In diesem Sinne sei hiermit eine erweiterte, ange-

19 Die Teildisziplin der Paläoanthropologie ist innerhalb der direkt folgenden Ausführungen völlig austauschbar mit der übergeordneten Disziplin der Paläontologie, denn in beiden Fällen bestehen weitgehend die selben prinzipiellen Problemstellungen. 
messenere Metapher aufgespannt: Als Grundlage dient die generelle Vorstellung eines zweidimensional auslegbaren Puzzles. Dieses wird jedoch nicht in einer originalen Verpackung mit Angaben und Garantien zum Inhalt erworben, sondern in Form eines Abonnements mit unterschiedlich großen Lieferungen, welche in unregelmäßigen Abständen ausgesendet werden. Es ist nicht bekannt, welche Größe bzw. Teilchenzahl das Puzzle letztendlich haben wird, und daher existieren auch keine als solche erkennbaren Randstücke. Darüber hinaus finden sich viele Puzzleteile doppelt, dreifach oder vielfach innerhalb der Gesamtheit der Lieferungen - und nicht nur sieht der aktuelle Zwischenstand noch große Lücken, sondern es ist tatsächlich nicht klar, ob letztendlich alle der zum Gesamtbild gehörenden Puzzleteile auch wirklich geliefert werden. In der Tat ist der aktuelle Zwischenstand noch in einem derart großen Umfang lückenhaft, sodass die Position vieler schon (teilweise) rekonstruierter Flecken des Puzzles nicht ganz geklärt ist. Mithin besteht mit jeder Lieferung die Gefahr, dass Teile des Puzzles umgelegt werden müssen - sowie dass sich damit die im gegebenen Moment geschätzte Größe des Puzzles als unzureichend erweist und erweitert werden muss.

Insgesamt erweist sich die soeben beschriebene Situation selbst für PuzzleEnthusiasten wohl als ein sehr abschreckendes Szenario. Dies entspricht der Wahrheit, obwohl längst nicht alle innerhalb der paläoanthropologischen Forschung gegebenen Problemstellungen Berücksichtigung fanden. So ist beispielsweise der Fall, dass Fossilfunde nicht zwingend sofort adäquat kategorisiert werden können. In diesem Sinne müsste im obigen Szenario eingeführt werden, dass innerhalb des Abonnements auch Puzzleteile geliefert werden, welche letztendlich gar nicht zum zu legenden Bild gehören, und welche daher erst durch vergleichende Untersuchung gewissenhaft aussortiert werden müssen - eine intuitiv praktisch unmögliche Aufgabe, solange das Gesamtbild noch nicht wenigstens im Groben feststeht. Ein weiteres Beispiel bezieht sich auf logistische Probleme der einschlägigen Forschung: Die im paläoanthropologischen Vorgehen entdeckten Fossilien werden nicht zentral, sondern in Einrichtungen der gesamten Welt eingelagert und konserviert, sodass ein einzelner Forscher sich weitgehend auf Beschreibungen und Abbildungen anderer Experten $\mathrm{zu}$ verlassen hat - dies jedenfalls, wenn kostspielige Weltreisen vermieden werden sollen.

Auf eine weitere Modifikation des obigen Szenarios soll verzichtet werden, denn es wurde bereits in einem angemessenen Maße verdeutlicht, wie die Metapher eines Puzzles selbst innerhalb des beschriebenen, durch Erweiterung an die Situation innerhalb der Paläoanthropologie angenäherten, fiktiven Puzzles es noch immer nicht vermag, die Realität angemessen abzubilden. Dies gilt in 
umso größerem Maße aufgrund der Tatsache, dass die Paläoanthropologie sich nicht mit einem einzelnen, scharfen, zweidimensionalen Bild beschäftigt, sondern mit einem Gesamtbild, welches einen nur schwer fassbar langen Zeitraum sowie ein geographisch außerordentlich weites Verbreitungsgebiet umfasst und welches die im vorigen Kapitel beschriebene evolutionäre Dynamik zur Grundlage hat - eine buschige, undurchsichtige Dynamik voller Trennungen, Sackgassen und Wiederzusammenführungen.

Alle diese Dimensionen erweitern und verschleiern die einem prototypischen Puzzle inhärenten scharfen Abgrenzungen und Erschweren eine Erarbeitung des endgültigen Gesamtbildes in einem derart großen Umfang, sodass die naive Verwendung des Attributs „komplex“ in keinster Weise zu vermitteln vermag, wie sehr die Paläoanthropologie mit ihrer inhärenten Unschärfe und mit ihren Schwierigkeiten sowie Limitationen zu kämpfen hat. Jahrzehnte der strukturierten Arbeit anhand tausender Funde kulminieren dennoch in einem durchaus gut belegten Szenario der Menschwerdung, welches nun folgend knapp skizziert werden soll.

Zugunsten der Übersichtlichkeit und Verständlichkeit sind die nachfolgenden Erläuterungen wiederum nicht als erschöpfend zu sehen, sondern erfüllen den Zweck einer einführenden und näherbringenden Skizze. Mitunter beinhaltet die einschlägige Forschung umfängliche Diskussionen zur Taxonomie der Gattung des Menschen - beispielsweise dazu, welche frühmenschliche Fossilfunde jeweils eigene Arten darstellen und welche $\mathrm{zu}$ gemeinsamen Arten $\mathrm{zu}$ sammengefasst werden sollten. Im Nachfolgenden wird zugunsten der Übersichtlichkeit gemeinhin so vorgegangen, dass die umfassender akzeptierte Interpretation unhinterfragt wiedergegeben wird. In besonders strittigen Fällen ist zwar dennoch eine entsprechende Anmerkung bzw. Klärung vorzunehmen, jedoch gemeinhin kann nicht auf jede offene Diskussion angemessen eingegangen werden. Für umfassendere Darstellungen der menschlichen evolutionären Linie siehe daher neben bereits genannten allgemeinen evolutionsbiologischen Einführungen Stringer \& Andrews (2005/2012), Tattersall (2015) und Dawkins \& Wong (2017, eine überarbeitete und erweiterte Neuveröffentlichung von Dawkins 2005) als stärker auf die Evolutionsgeschichte des Menschen fokussierte populäre Veröffentlichungen.

\subsection{Vom letzten gemeinsamen Vorfahren zu den Australopithecinen}

Der Weg spezifisch menschlicher Evolution beginnt freilich beim letzten gemeinsamen Vorfahren zwischen dem Menschen und seinen nächsten Verwand- 
ten - den beiden rezenten Arten der Gattung Pan, also dem Gemeinen Schimpansen (Pan troglodytes) und dem Bonobo (Pan paniscus). Eine genaue zeitliche Verortung dieses letzten gemeinsamen Vorfahren gestaltet sich selbst innerhalb der jüngeren Forschung noch als schwierig, da im Sinne der bereits beschriebenen, höchst dynamischen evolutionären Dynamik keine plötzliche, vollständige Trennung zweier Linien gegeben war. Stattdessen bestanden über längere Zeiträume wiederkehrende unvollständige Trennungen und wiederholter Genfluss, bevor die beiden Linien hinreichend getrennt waren, um eine Fortpflanzung vollständig auszuschließen (vgl. bspw. Patterson et al. 2006 einschließlich der knappen Kommentare bzw. Nachträge durch Wakeley 2008 und Patterson et al. 2008).

In diesem Sinne finden sich in der einschlägigen Literatur je nach methodischem Vorgehen stark abweichende Hinweise auf die Trennung der beiden taxonomischen Gruppen. Arnason, Gullberg \& Janke (1998) sowie Arnason et al. (2000) liegen mit einer Datierung auf vor etwa 10-13 Millionen Jahren auf der einen Seite des Spektrums und Adachi \& Hasegawa (1995), Takahata \& Satta (1997) sowie Stone et al. (2010) finden sich mit einer Datierung auf etwa 4-5 Millionen Jahre auf der anderen Seite des Spektrums. Das online verfügbare Tool Timetree (Kumar et al. 2017), welches mehr als 70 relevante Studien katalogisiert und einen Median der jeweiligen Ergebnisse inklusive Fehlerbalken zur Verfügung stellt, setzt als kumulatives Ergebnis 6,2-7,1 Millionen Jahre als wahrscheinlich fest. Genetische Studien der 90er Jahre und des frühen neuen Jahrtausends bewegen sich dabei meist im Bereich von 5-7 Millionen Jahren, während jüngere Veröffentlichungen dagegen insbesondere im Hinblick auf neuere fossile Funde zu einer zeitlichen Verortung von vor wenigstens etwa 7 Millionen Jahren tendieren, wobei ein Beginn des Trennungsprozesses gelegentlich auf bis vor etwa 10 Millionen Jahren verortet wird (vgl. bspw. White et al. 2009 sowie Langergraber et al. 2012).

Mit diesen Daten als Kontext ist hiermit auf Sahelanthropus tchadensis zu verweisen - ein vorgeschlagenes Taxon $\mathrm{zu}$ einem bedeutenden Fossilfund, welcher nach aktueller Einschätzung eine große Nähe zum letzten gemeinsamen Vorfahren des Menschen mit dem Schimpansen aufweist und auf etwa 7 Millionen Jahre datiert wird (vgl. zur morphologischen Einschätzung und Datierung Guy et al. 2005, Wolpoff et al. 2006 und Lebatard et al. 2008). Auf Basis der Feststellung, dass dieses Taxon möglicherweise bereits eine nennenswerte Befähigung zu einer aufrechten Haltung aufwies - jedenfalls im Vergleich mit Schimpansen - , und da örtlich weit entfernte, jedoch evolutionär anscheinend in nennenswertem Maße nah verwandte Funde eine ähnlich große bzw. teils sogar größere Befähigung zur aufrechten Haltung bzw. Fortbewegung aufwei- 
sen (vgl. bspw. Fuss et al. 2017, Böhme et al. 2017 und Gierliński et al. 2017 zu jüngeren Entdeckungen, Rook et al. 1999 zu länger bekannten Funden), sind zwei Schlussfolgerungen naheliegend:

Erstens besteht hierdurch ein Hinweis auf eine oder mehrere umfangreiche adaptive Radiation(en) in verwandtschaftlicher Nähe zum genannten Sahelanthropus tchadensis bzw. hinsichtlich der damaligen Vorfahren heutiger Menschenaffen generell. Anscheinend waren bis $\mathrm{zu}$ jenem Zeitpunkt Menschenaffen wesentlich weiter verbreitet als sie es - den Menschen dabei ausgenommen - heute sind (bspw. Rook et al. 1999, Heizmann \& Begun 2001, DeMiguel, Alba \& Moyà-Solà 2014 und Fuss et al. 2017). Günstige, subtropische klimatische Bedingungen sowie eine dichte Bewaldung erstreckten sich nicht nur großflächig in Afrika, sondern führten Arten mit hominiden Eigenschaften bis nach Südostasien, wo sie unter anderem die Linie bis zu heutigen Populationen der Orang-Utans begründeten, und Europa - ein Umstand, der dadurch, dass das Mittelmeer zeitweise austrocknete und damit einen direkten Landweg bot, zusätzlich unterstützt wurde (vgl. bspw. Krijgsman et al. 2001).

Zweitens ist naheliegend, dass der Mensch nicht aus einer dem Schimpansen morphologisch sehr ähnlichen Art entstanden ist, sondern dass sowohl der Schimpanse als auch der Mensch von einem Wesen abstammen, das bereits eine zum aufrechten Gang begünstigende Morphologie des Schädelansatzes, des Rückgrats, des Beckens und weiterer körperlicher Strukturen aufwies. Der evolutionäre Weg des Schimpansen hin zu seiner heutigen Fortbewegungsweise und die Veränderung der zugehörigen Morphologie ist daher als wenigstens ebenso umfangreich anzusehen wie die des Menschen und entwickelte sich unabhängig von der ähnlichen Fortbewegungsweise des modernen Gorillas (Kivell \& Schmitt 2009).

Als Ursprung dieser teilweisen Befähigung zum aufrechten Gang beim gemeinsamen Vorfahren wird gemeinhin ein Lebensstil angesehen, welcher die Bewohnung von Bäumen zeitlich umfassend beinhaltete (bspw. White et al. 2009, Kivell \& Schmitt 2009 sowie Lovejoy et al. 2009a und 2009b). Ebendieser Lebensstil brachte einen relativ geraden Anschluss des Rückgrats an den Schädel sowie weitere relevante Anpassungen mit sich - weswegen es beispielsweise auch der Fall ist, dass ein aufrecht gehender Orang-Utan einem Menschen in dieser Hinsicht ähnlicher wirkt als die mit dem Menschen näher verwandten, zum Knöchelgang angepassten Gorillas und Schimpansen bei deren fakultativ aufrechter Fortbewegung (vgl. Thorpe et al. 2007 trotz der Kritik durch Begun, Richmond \& Strait 2007).

Dieser Ausgangslage um Sahelanthropus tchadensis folgt eine nur äußerst lückenhaft belegte Entwicklung hin zu einer zunehmend aufrechten, bodenna- 
hen Lebensweise und einer entsprechenden morphologischen Anpassung, welche jedoch anscheinend nur langsam geschah und daher darauf hinweist, dass der Übergang zum aufrechten Bodenbewohnen innerhalb dieser und folgender Arten bzw. Gattungen weder plötzlich noch vollständig geschah. Zu erwähnen sind in diesem Kontext Orrorin tugenensis (Senut et al. 2001 sowie Richmond \& Jungers 2008 und Almécija et al. 2013) sowie die Gattung Ardipithecus mit den beiden Arten Ardipithecus ramidus (White, Suwa \& Asfaw 1994 und 1995 sowie White et al. 2009) und Ardipithecus kadabba (Haile-Selassie, Suwa \& White 2004), welche allesamt wenigstens zum allgemeinen Formenkreis, welcher die Linie hin zum Menschen beinhaltet, zu zählen sind, dabei jedoch möglicherweise bzw. wahrscheinlich nicht innerhalb der tatsächlichen Linie zum Menschen auffindbar sind. Die erstgenannte Art datiert auf vor etwa 6 Millionen Jahren und die Gattung Ardipithecus ist zeitlich auf vor etwa 5,8 bis 4,4 Millionen Jahre zu verorten - was eine große zeitliche Nähe mit den Australopithecinen bedeutet, welche gemeinhin als Teil der direkten evolutionären Linie hin zur Gattung des Menschen angesehen werden.

\subsection{Von den Australopithecinen zur Gattung des Menschen}

Ebendieser Gattung Australopithecus, welche auf ab vor etwa 4,2 Millionen Jahren verortet wurde (Patterson, Behrensmeyer \& Sill 1970 und Ward, Leakey \& Walker 2001), ist eine große Bedeutung innerhalb der Paläoanthropologie zuzurechnen. Dies rührt einerseits davon her, dass erstmals eine verhältnismäßig große Zahl von Funden zu einer Reihe von Arten (A. afarensis, A. africanus, $A$. anamnensis, A. garhi neben Weiteren) vorliegt, womit eine umfangreichere Rekonstruktion weiterer evolutionärer Veränderungen ermöglicht wird, und basiert andererseits darauf, dass sich in zunehmendem Maße evolutionäre Anpassungen finden, welche als typisch für die menschliche Linie anzusehen sind. In diesem Sinne finden sich bei Australopithecus Anpassungen der Wirbelsäule, des Beckens, der Hüftgelenke, der Hände, der Füße und des Gebisses nebst weiteren Veränderungen, welche als zum aufrechten Gang sowie zu einer omnivoren Ernährungsweise und zur Handhabung von Werkzeugen richtungsweisend anzusehen sind (Latimer \& Lovejoy 1989, Alba, Moyà-Solà \& Köhler 2003, Raichlen et al. 2010, McPherron 2010, Kivell et al. 2011 und Kozma et al. 2018). Dies gilt, obwohl Australopithecus sich vermutlich noch immer ausgiebig in Bäumen aufhielt (Green \& Alemseged 2012). Spätere Australopithecine sowie Kenyanthropus platyops (Leakey et al. 2001 und Liebermann 2001), dessen Rolle in Bezug auf die Evolutionsgeschichte des Menschen noch nicht abschließend geklärt ist, weisen weitere Anpassungen auf, inkorporierten in ihre Lebensweise 
anscheinend einen opportunistischen, möglicherweise Aas umfangreich enthaltenden, Fleischkonsum (Domínguez-Rodrigo et al. 2005 sowie McPherron et al. 2010) und verwendeten erstmals eine äußerst simple Form bearbeiteter Steinwerkzeuge (nebst Vorgenannten auch Harmand et al. 2015 zu dieser vorbehaltlich als „Lomekwi-Kultur“ bezeichneten Werkzeugtradition).

Hier ist sinnvoll, einen kleinen Exkurs einzuschlagen, denn diese Entwicklung gilt primär für eine von zwei groben Tendenzen, die sich innerhalb der Gattung Australopithecus finden. Im Verlauf der evolutionären Entwicklung der Gattung kam es zur morphologischen Herausbildung eines Formenkreises, dem „robuste“ Arten zugehörten, sowie einer davon als „grazile“ Australopithecine abgegrenzten Artengruppe. Die morphologischen Unterschiede bestanden dabei primär in der Größe der Zähne, des Gebisses, der Kiefer- bzw. Kaumuskulatur sowie der allgemeinen Kopf- und Stirnform (vgl. bspw. Constantino 2013 und Fleagle 2013). Jene robusten Australopithecine existierten als eine Schwesterngruppe zur Gattung des Menschen und stellten im Kontext eines klimatischen Langzeittrends zur größeren Trockenheit im Verbreitungsgebiet der Gattung eine Anpassung zur zunehmend pflanzlichen Ernährung dar (Cerling et al. 2011 und Bibi et al. 2012) - im Gegensatz zur sehr auf Früchte orientierten Diät früherer Arten des Formenkreises und auch wenn anscheinend kein vollständiger Wechsel zur alleinigen Ernährung über Gräser geschah, wie in der Vergangenheit vorgeschlagen wurde (Martínez et al. 2016). Jene robusten Australopithecinen werden ab vor etwa 2,5 Millionen Jahren in Abgrenzung zur Gattung des Menschen, Homo, innerhalb der Schwesterngattung Paranthropus zusammengefasst. Im Gegensatz zur menschlichen Linie konnte zu den Arten jener Gattung die Verwendung von Steinwerkzeugen nicht eindeutig nachgewiesen werden und es zeigen sich auch keine nennenswerten Umstellungen im Sozialverhalten und - verglichen mit den früheren Australopithecinen - keine bedeutsame Vergrößerung des Gehirnvolumens trotz einer durchaus ausgeprägten Befähigung zum aufrechten Gang und morphologisch relativ moderner Hände (Macchiarelli et al. 1999, Wood \& Richmond 2000, Grine et al. 2012 sowie Susman 1988).

Im Gegensatz zu jenen robusten Australopithecinen, welche in der inzwischen ausgestorbenen Gattung Paranthropus mündeten, zeigten manche der grazilen Australopithecinen eine Reihe von Anpassungen, welche die Gattung des Menschen prototypisch bestimmen: Zum einen zeigt sich die verstärkte Tendenz zu einer omnivoren Ernährung mit einem zunehmenden Fleischkonsum mithilfe der sich ausprägenden Steinwerkzeugkultur des Oldowan (Semaw 2000, Domínguez-Rodrigo et al. 2005, McPherron et al. 2010, Macho 2014 und Pobiner 2013) und zum anderen zeigen sich Veränderungen im Sozialverhalten 
- eine primäre Schlussfolgerung aus der Reduktion des Geschlechtsdimorphismus innerhalb der einschlägigen Arten. Diese Tendenz zu einem variableren Verhalten sowie zur veränderten und gesteigerten Soziabilität nebst Sozialität scheint sich im weiteren evolutionären Verlauf fortzusetzen und lässt sich innerhalb Homo schließlich umfangreich belegen. Dazu gehören in letzter Konsequenz auch Funde, welche eine gesteigerte Kooperationsfähigkeit sowie die zunehmende Ausbildung von altruistischem Verhalten stark nahelegen (McHenry 1992 und 1994, Lordkipanidze et al. 2005, Bonmatí et al. 2010, Burkart et al. 2014, Trinkhaus \& Villotte 2017 und etwas allgemeiner Hublin 2009). ${ }^{20}$

Damit besteht ein thematischer Übergang zur Gattung des Menschen: Während die Australopithecinen zwar bereits eine sukzessive Anpassung bzgl. der Morphologie ihrer Beine, Arme, Zähne, Hüfte sowie Schultern zeigten und wenigstens innerhalb der grazilen Tradition - einen opportunistischen Fleischkonsum sowie die Verwendung simpler Steinwerkzeuge in ihre Überlebensstrategien implementierten, so ist keine bzw. nur wenig Veränderung hinsichtlich anderer Aspekte festzustellen. Dazu gehört die kaum veränderte Körpergröße sowie ein weitgehend stagnierendes Volumen des Gehirns, welche sich beide noch in etwa vergleichbar mit dem modernen Schimpansen sowie mit dem gemeinsamen Vorfahren zwischen dem Menschen und dem Schimpansen zeigen. ${ }^{21}$ Innerhalb einer oder mehrerer adaptiven Radiationen entstehen jedoch ab vor etwa 2,5 Millionen Jahren erste Arten, welche gemeinhin der Gattung des Menschen zugeschrieben werden, da sie nebst einer Weiterführung bestehender Tendenzen zusätzlich genau jene prototypisch menschlichen Anpassungen zeigen, welche in Australopithecus noch nicht zu finden sind.

20 Die hier lediglich unspezifisch über den Verweis auf die jeweiligen Veröffentlichungen erwähnten Beispiele werden zu einem späteren Zeitpunkt erläutert und diskutiert.

21 Das Gehirnvolumen von Schimpansen, dem gemeinsamen Vorfahren sowie der menschlichen Linie bis zur Endphase der Australopithecinen ist auf grob $400 \mathrm{~cm}^{3}$ anzusetzen. Erst diejenigen Australopithecine, welche dem Übergang zur Gattung des Menschen direkt anschließen, zeigen immerhin ansatzweise eine Vergrößerung bis auf etwa $500 \mathrm{~cm}^{3}$ (vgl. Lockwood et al. 1999 sowie Seymour et al. 2016). Dies mit der Anmerkung, dass es innerhalb einzelner Arten zu durchaus großer individueller Variation kommt und so auch Schimpansen in Ausnahmefällen bis zu $500 \mathrm{~cm}^{3}$ Gehirnvolumen erreichen können. 


\subsection{Die frühen und mittleren Formen der Gattung des Menschen}

So besitzt $H$. rudolfensis ${ }^{22}$ bei einer noch nicht besonders veränderten Körpergröße bereits ein nennenswert gesteigertes Gehirnvolumen von etwa 700-750 $\mathrm{cm}^{3}$ und eine besonders auffällige Anpassung der Kopfform und des Gesichts in Richtung Modernität (Bromage et al. 2008 sowie Seymour et al. 2016). Weitere Arten des Formenkreises dieser Frühphase der Gattung Homo von vor etwa 2,5 Millionen Jahren bis vor etwa 1,8 Millionen Jahren - dazu gehören nebst dem genannten $H$. rudolfensis insbesondere auch $H$. habilis, $H$. ergaster und $H$. georgicus - zeigen zwar ein zueinander abweichendes morphologisches Mosaik hinsichtlich konkreter Eigenschaften, jedoch zugleich eine vergleichbare, allgemeine Tendenz zum vergrößerten Gehirnvolumen sowie zur jeweiligen Herausbildung eines Teils der typisch menschlichen körperlichen Eigenschaften, womit die Wildheit und die sukzessive sowie mosaische Natur der stattfindenden adaptiven Radiation(en) nochmals unterstrichen wird (Leakey et al. 2012, Lordkipanidze et al. 2013, Kimbel \& Villmoare 2016 und Seymour et al. 2016).

Eine zwischenzeitliche Kulmination erreicht diese Entwicklung mit der Art $H$. erectus, zu welcher die vorgenannten $H$. ergaster sowie $H$. georgicus in der einschlägigen Literatur teils als Chronospezies und teils als lokale Varietäten angesehen werden (Vekua et al. 2002, Antón 2003 und Lordkipanidze et al. 2007 nebst Lordkipanidze et al. 2013). Unabhängig von der jeweiligen Interpretation ist jedoch festzustellen, dass $H$. erectus einen zwischenzeitlichen Meilenstein darstellt, da erstmals eine größere Summe relativ moderner anatomischer Eigenschaften innerhalb einer einzelnen Art zusammenkommen - einer hinsichtlich ihres Verbreitungsgebiets, ihrer Langlebigkeit und ihrer technologischen sowie kognitiven Entwicklung äußerst erfolgreichen Art, wie nachfolgend knapp skizziert werden soll.

H. erectus bzw. sein näherer Formenkreis etablierte sich im Rahmen seines Aufkommens vor etwa 1,9 Millionen Jahren erstaunlich plötzlich nicht nur in Afrika, sondern auch im Nahen Osten sowie in größeren Teilen Ostasiens und Südostasiens (Bar-Yosef \& Belfer-Cohen 2001, Rightmire 2001, Zhu et al. 2008, Bettis, III et al. 2009, Garcia et al. 2010 und Ferring et al. 2011). ${ }^{23}$ Mit Verzöge-

22 Heute meist als eine eigene Art angenommen trotz durchaus bestehender Kontroverse (vgl. Wood 1999 sowie Wood \& Richmond 2000).

23 Eine Studie von Zhu et al. (2018) suggeriert sogar eine entsprechende Etablierung und Ausbreitung vor mehr als 2 Millionen Jahren, wobei deren Ergebnisse ohne weitere bestätigende, triangulierende Veröffentlichungen mit Vorbehalt angenommen werden sollten. 
rung geschah schließlich auch die Besiedelung Europas vor etwa 1,4 Millionen Jahren (vgl. nochmals Bar-Yosef \& Belfer-Cohen 2001). Es gibt abweichende Meinungen darüber, ob diese recht plötzliche Ausbreitung im Rahmen eines aktiven, demographisch begründeten Verhaltens geschah (Carbonell et al. 2010) oder ob allein passive Überlebensstrategien als eine hinreichende Erklärung dieser Entwicklung ausreichen (Carotenuto et al. 2016), jedoch ist der generelle Erfolg der Art darauf zurückzuführen, dass sie bezüglich Technologien, Verhaltensstrategien und gesteigerter Soziabilität eine Leistungsfähigkeit erreichten, wie sie zuvor innerhalb der Gattung des Menschen anscheinend nicht gegeben war.

So entwickelte sich relativ bald nach Aufkommen und Verbreitung dieses Formenkreises eine im Vergleich $\mathrm{zu}$ bestehenden Steinwerkzeugen der Oldowan-Kultur qualitativ komplexere Werkzeugtradition des Acheuléen (Lepre et al. 2011), welche sich anschließend sukzessive verbreitete und nach einem langwierigen Verdrängungsprozess weltweit gegenüber den OldowanWerkzeugen durchsetzte (Goren-Inbar et al. 2000 sowie Scott \& Gibert 2009). Gegenüber der Feststellung, dass sich bereits bei den Australopithecinen ein opportunistischer Fleischkonsum einstellte, bleibt $\mathrm{zu}$ bemerken, dass in den hier gegebenen Zeitrahmen erstmals ein aktives und regelmäßiges Jagen $\mathrm{zu}$ fallen scheint (Gaudzinski 2004, Rabinovich, Gaudzinski-Windheuser \& GorenInbar 2008, Ferraro et al. 2013), möglicherweise bereits teilweise in Form von Ausdauerjagd (Bramble \& Liebermann 2004 sowie Liebermann et al. 2007). Dies wird durch die Feststellung, dass Frühmenschen ab vor grob zwei Millionen Jahren begannen, ihre ökologische Umwelt wesentlich freier und variabler $z u$ nutzen als es in vorhergehenden Arten gegeben war, weiter unterstützt (vgl. Plummer et al. 2009).

Trotz dieser generellen Variabilität bilden sich großflächig erstaunlich homogene und effiziente Beschaffungsstrategien heraus (Pobiner et al. 2008), womit ein Hinweis auf eine leistungsfähige kulturelle Tradierung dieser Strategien gegeben ist. In anderen Worten: Während die Australopithecinen sowie die frühesten menschlichen Arten sich an ihre angestammten, prototypischen Habitate hielten, stellte sich nun ein Lebensstil ein, der eine Vielzahl natürlicher Lebensräume - von offenem Grasland über Bewaldung zu kargen Hügelregionen und Küstenregionen sowie Feuchtgebieten - umfasste und so von einer zunehmend variablen sowie zunehmend leistungsfähigen Überlebensstrategie zeugt. Dem allem sind die vormals bereits erwähnten Funde, welche eine Herausbildung gesteigerter Kooperationsfähigkeit nebst altruistischem Verhalten nahelegen und in diesen Zeitraum großer Umstellungen innerhalb der Gattung Homo fallen, hinzuzustellen. 
Dazu gehört der Fund von 1,5 Millionen Jahren alten Fußspuren, welche von wenigstens 20 Individuen stammen und eine geschlossene, koordinierte Fortbewegung einer Gruppe mit großem Männeranteil nahelegen (Hatala et al. 2016). Sozial lebende Arten der höheren Säugetiere, in denen ein großer Geschlechtsdimorphismus festgestellt werden kann, haben typischerweise eine soziale Struktur, in welcher ein dominantes Männchen über einen Harem verfügt, in dem weitere Männchen nur als Jungtiere bzw. als periphere Mitglieder der Gruppe toleriert werden - d.h. der aggressive Wettbewerb zwischen Männchen bietet einen großen evolutionären Selektionsdruck (Gordon 2006), wodurch ebenjener Geschlechtsdimorphismus zu erklären ist. Alle Vorfahren der Gattung des Menschen einschließlich weitestgehend den direkt vorhergehenden Australopithecinen sowie auch die Schwesterngattung des Menschen, Paranthropus, zeigen noch einen derartigen großen Geschlechtsdimorphismus und dementsprechend eine sehr wahrscheinlich vergleichbare soziale Struktur (vgl. bspw. McHenry 1994, Richmond \& Jungers 2008, Gordon et al. 2008 sowie Grine et al. 2012 trotz Kontroverse zum Ausmaß des Geschlechtsdimorphismus in Australopithecus bspw. bei Reno et al. 2003 sowie Reno \& Lovejoy 2015). Im weiteren evolutionären Verlauf der späteren grazilen Australopithecinen sowie insbesondere innerhalb der Gattung Homo löst sich dieser Geschlechtsdimorphismus jedoch sukzessive auf, sodass bis zur aktuell beschriebenen Situation rund um $H$. erectus in dieser Hinsicht eine näherungsweise moderne Konstellation erreicht wird (Garvin et al. 2017). Dies, gepaart mit Hinweisen wie den soeben erwähnten Fußspuren, lässt den Schluss auf große Umstrukturierungen im Sozialverhalten $\mathrm{zu}$ - vermutlich einschließlich gesteigerter Kooperationsfähigkeit.

Hinzu kommen weitere Funde, die zusätzlich altruistisches Verhalten in Form von sozialer Fürsorge nahelegen. Vormals erwähnte Veröffentlichungen (McHenry 1992 und 1994, Lordkipanidze et al. 2005, Bonmatí et al. 2010, Burkart et al. 2014, Trinkhaus \& Villotte 2017 und Hublin 2009) beschreiben eine Reihe von fossilen Funden, in denen Individuen starken gesundheitlichen Einschränkungen ausgesetzt worden waren, diese jedoch teils um Jahre und in ein hohes Alter überlebten (vgl. auch Spikins et al. 2018: 11). Obwohl durchaus spekulativ, so ist gemäß den außerordentlichen Einschränkungen, welche jene Verletzungen bzw. Erkrankungen - u.a. beinahe vollständige Zahnlosigkeit, starke Verknöcherung von Gelenken mit großen Bewegungseinschränkungen und schwere Frakturen verschiedener Körperteile einschließlich des Kopfes - mit sich bringen, dennoch naheliegend, dass ein Überleben ohne soziale, altruistische Intervention nicht oder nur äußerst erschwert möglich gewesen wäre. Die Schlussfolgerung ist damit gemeinhin, eine entsprechend stark unterstützende 
soziale Struktur zu unterstellen - eine stark unterstützende soziale Struktur, welche vermutlich auch Kooperation im Kontext der Versorgung des Nachwuchses mit einbezieht (van Schaik \& Burkart 2010, Vaesen 2012).

Schließlich erreichte $H$. erectus im evolutionären Verlauf seines Formenkreises ein durchaus erstaunliches Gehirnvolumen, welches bis in die unteren Bereich der typischen Variation des modernen Menschen reicht (Rightmire 2004), auch wenn das entsprechende ontogenetische Wachstum in jener Art wohl kürzer ausfiel als im modernen Menschen (Leigh 2006), was die kognitive Entwicklung womöglich begrenzte. Zugleich stieg die Intensität der Blutversorgung des Gehirns des $H$. erectus ebenfalls bedeutend an - und verglichen mit dem Zuwachs des Gehirnvolumens unverhältnismäßig stark und früh - , was für eine dichtere und leistungsfähigere neuronale Vernetzung spricht (Seymour et al. 2016). Insgesamt kann so unter Einbezug der archäologisch-kulturellen Funde wohl durchaus von einer nennenswerten kognitiven Leistungsfähigkeit ausgegangen werden, auch wenn dabei sicherlich nicht von Modernität ausgegangen werden sollte.

Für grob eine Million Jahre nach Aufkommen und Verbreitung von $H$. erectus bzw. des zugehörigen näheren Formenkreises nahm dieses Taxon im Sinne des soeben Erläuterten innerhalb der Gattung des Menschen eine zentrale Rolle ein. In Abgrenzung zur vormals spezifizierten Frühphase der Gattung von vor 2,5 bis vor 1,8 Millionen Jahren kann hier damit von einer mittleren Phase spezifisch menschlicher Evolution gesprochen werden. Dies gilt nicht nur für technologisch-kulturelle sowie ökologische Aspekte, sondern begründet sich auch darin, dass weitere Radiationen und physiologische Entwicklungen in Richtung Modernität maßgeblich innerhalb dieses Formenkreises geschahen, obwohl weitere Arten der Gattung Homo weiterhin parallel existierten, wie beispielsweise durch überraschend junge Funde des vergleichsweise archaischen $H$. naledi verdeutlicht wird (Hawks et al. 2017 sowie Berger et al. 2017). Zuletzt macht diese Interpretation dahingehend Sinn, dass innerhalb dieses Zeitraums trotz sukzessiver weiterführender Entwicklungen keine qualitativ maßgebende Zäsur feststellbar ist - jedenfalls nicht bis zum Aufkommen der Art H. heidelbergensis und dem Beginn der frühen Spätphase.

\subsection{Weitere Radiationen in Richtung Modernität und frühe kumulative Kultur}

$H$. heidelbergensis stellt vermutlich das Ergebnis einer oder mehrerer weiterer Radiationen aus dem Formenkreis um $H$. erectus dar, kommt in früher prototypischer Form vor etwa 850.000 Jahren in Afrika auf und etabliert sich bis vor 
grob 700.000 Jahren großflächig und in einem wiederum anscheinend überraschend kurzen Zeitrahmen in Europa und Afrika sowie möglicherweise bzw. vermutlich Teilen Asiens (Rightmire 1998, Mounier 2009, Stringer 2012, Buck \& Stringer 2014 sowie Profico et al. 2016). ${ }^{24}$ Dem ist $\mathrm{zu}$ folgern, dass H. heidelbergensis - mehr noch als bei früheren Radiationen des Formenkreises - sich äußerst schnell gegenüber bestehenden Populationen durchsetzen konnte und sich fortan in weiten Teilen Afrikas, Europas und vermutlich Asiens als dominierend zeigte. Dies korreliert mit qualitativen technologischen Neuerungen, weiteren physiologischen Entwicklungen sowie das Verhalten betreffende Umstellungen.

Hierzu ist mitunter eine freie Kontrolle über Feuer zu zählen. Zwar ist eine opportunistische Nutzung von Feuer möglicherweise ein durchaus archaisches, wenigstens 1,5 Millionen Jahre altes Verhalten, jedoch ist die freie Schaffung und Kontrolle von Feuer als ein vergleichsweise jüngerer Meilenstein zu zählen - ein Meilenstein, der vermutlich in den Kontext von $H$. heidelbergensis ${ }^{25}$ sowie potenziell mit einem Alter von bis zu 800.000 Jahren zu verorten ist (GorenInbar et al. 2004; Gowlett 2016), auch wenn sich ein derartiges Verhalten laut der aktuellen Fundlage erst vor etwa 400.000 Jahren großflächig und mit klarer Persistenz durchsetzen konnte.

Mit jenem Datum von etwa 400.000 Jahren sind auch Funde von Speerwaffen, deren Spitzen mit Teer verklebt wurden - ein technologischer Vorgang, für den große Hitze und damit Feuer vonnöten war (Thieme 1997, Roebroeks \& Villa 2011) - und welche jenen Frühmenschen mittels dieser Wurfwaffen möglicherweise das Spektrum der Jagdstrategien moderner Jäger und Sammler eröffneten (Schoch et al. 2015, Milks, Parker \& Pope 2019), ${ }^{26}$ sowie die ältesten Hinweise auf simple Behausungen (Roebroeks \& van Kolfschoten 1994) und eine frühe

24 Es ist darauf hinzuweisen, dass sich ein erweiterter morphologischer Formenkreis möglicherweise bereits vor etwas mehr als einer Million Jahren herausbildete und einige physiologische Eigenschaften des $H$. heidelbergensis vorwegnahm - ein Vorgang recht ähnlich des bereits Beschriebenen im Kontext der Frühphase des Menschen hin zur zwischenzeitlichen Kulmination in $H$. erectus. In diesem Sinne wäre die Art $H$. heidelbergensis taxonomisch weiter zu fassen und zeitlich älter als jenseits dieser Fußnote dargestellt wird (vgl. Mounier et al. 2011). In diesem Sinne könnte nebst weiteren Arten auch $H$. antecessor hinzuzustellen sein (Bermúdez de Castro et al. 1997 sowie 2017). Die jenseits dieser Fußnote beschriebene Situation stellt jedoch den gängigen Konsens im Sinne einer engeren Interpretation der Art dar.

25 Möglicherweise sind Funde zu Feuerstellen innerhalb dieses zeitlichen Rahmens in China der dortigen Variante von $H$. erectus zuzuordnen (Gowlett 2016).

26 Faivre et al. (2019) weisen in verwandter Sache skelettale Abnormalitäten bei NeandertalerIndividuen nach, welche mit den körperlichen Verschleißerscheinungen heutiger professioneller Wurfathleten übereinzustimmen scheinen. 
Verwendung von Farbe (Barham 2002) verbunden. Zusätzlich zu nennen sind auch archaische Formen einer von früheren Steinwerkzeugen qualitativ abzugrenzenden Werkzeugtradition, der Levalloistechnik (Teil der Wegbereitung der späteren Moustérien-Kultur; Foley \& Lahr 1997), welche in ihrer Herstellung eine hohe kognitive Leistungsfähigkeit voraussetzen - d.h. eine Befähigung zur Planung und Durchführung entlang einer komplexen, hierarchischen Informationsstruktur (Eren \& Lycett 2012). Die Herstellung dieser Werkzeuge an geeigneten regionalen Werkstätten erreichte dabei mitunter solche Ausmaße, dass die örtliche Ökologie durch die umfangreiche Produktion von Abschlägen abgeändert wurde (Foley \& Lahr 2015).

Die vorgenannten Werkzeuge und Verhaltensweisen sowie die scheinbar bewusste Entsorgung von Toten im Rahmen von frühem begräbnisartigem Verhalten (Arsuaga et al. 1997 sowie Aranburu, Arsuaga \& Sala 2017) suggerieren eine sehr komplexe mentale Welt bei diesen Frühmenschen, welche möglicherweise ein bereits recht angenähertes Kontinuum mit den späteren Populationen des Neandertalers sowie des anatomisch modernen Menschen nebst den Denisova-Menschen bilden, wie Eren \& Lycett (2012) anhand des Vergleichs von Steinwerkzeugen nahelegen. Auch Hosfield \& Cole (2018) stellen auf Basis archäologischer Funde einen nennenswerten technologisch-kulturellen, d.h. kognitiven, Bruch mit der bisherigen Tradition vor grob 800.000 bis 600.000 Jahren fest. Jene Autoren argumentieren - auch im Sinne von Powell, Shennan \& Thomas (2009) - darüber hinaus, dass ab dieser Stufe menschlicher Evolution demographische Entwicklungen möglicherweise mehr Einfluss auf Kultur und Technologien gehabt haben könnten als biologisch begründete kognitive Entwicklungen: „[O]ur results are [...] suggesting that cognitive capacities in different species of Middle-Late Pleistocene hominins are not as sharply differentiated as previous generations of scholars postulated“ sowie „[B]ehavioural changes that eventually emerge [...] may be more the product of demographic change and increased connectivity of social networks than they were, necessarily, of fundamental cognitive changes“ (beide Eren \& Lycett 2012: 9).

Diese augenscheinlich bereits in vielerlei Hinsicht weit entwickelte Art des Homo heidelbergensis scheint schließlich der Ausgangspunkt zu lokalen Entwicklungen zu sein (Rogers, Bohlender \& Huff 2017), welche die Spätphase der menschlichen Evolution darstellen. In Afrika führt eine derartige Entwicklung im Rahmen mehrerer Populationen innerhalb eines gemeinsamen Formenkreises - unter anderem dabei vermutlich über $H$. rhodesiensis - zum anatomisch modernen Menschen im Rahmen eines geographisch großflächigen Verbreitungsgebiets sowie innerhalb eines komplexen adaptiven Mosaiks, das noch nicht vollständig rekonstruiert ist und in umfänglichen Teilen der einschlägigen 
Literatur schlicht unter dem Deckmantel des $H$. heidelbergensis zusammengefasst wird (Rightmire 1983, Mounier et al. 2011, Hublin et al. 2017 sowie Stringer \& Galway-Witham 2017).

In Europa entwickelt $H$. heidelbergensis im Rahmen seiner lokalen Entwicklung bereits vor etwa 400.000 Jahren prototypische Eigenschaften des späteren Neandertalers heraus und gilt daher mit vergleichsweise großer Sicherheit als zentral in der Linie hin zu jener späteren Schwesternart des anatomisch modernen Menschen (Arsuaga et al. 2014 sowie 2015, Meyer et al. 2016). Zu betonen ist hierzu, dass im Rahmen der aktuellen Forschungslage hinsichtlich Technologien, Verhaltensstrategien und sozialer Strukturen innerhalb dieses evolutionären Verlaufs kein nennenswerter qualitativer Unterschied zwischen den europäischen und den afrikanischen Populationen festgestellt werden kann, wie im Weiteren noch knapp dargestellt werden soll. Dieser Befund ist als bedeutend anzusehen, weil er dem Konsens mehrerer Generationen paläoanthropologischer Forschung widerspricht und dennoch die jüngere Forschungslandschaft zunehmend bestimmt. Eine Erklärung und Erläuterung dieses Paradigmenwechsels wird in einem Kapitel des Analyseteils vorgestellt werden, um die dortige Argumentation zusätzlich zu stützen.

In weiten Teilen Kontinentalasiens sowie im südostasiatischen Raum scheint $H$. heidelbergensis schließlich die zu den Neandertalern sowie zum modernen Menschen als eine weitere Schwesternart einzuordnende Population der Denisova-Menschen zu begründen (Krause et al. 2010, Reich et al. 2010, PaixáoCôrtez et al. 2012), deren Existenz ebenfalls als die jüngere Forschungslage maßgeblich bestimmend anzusehen ist. Da die paläoanthropologische und archäologische Fundlage hier jedoch vergleichsweise äußerst gering ausfällt, basieren einschlägige Schlussfolgerungen zentral auf genetischen Untersuchungen sowie auf nicht ohne Spekulation auskommenden Vergleichen mit der afrikanischen und europäischen Entwicklung.

\subsection{Variables und symbolisches Verhalten in der Spätphase menschlicher Evolution}

Spätestens mit dem Aufkommen des anatomisch modernen Menschen bis vor grob 200.000 Jahren ist die Spätphase der Menschwerdung wohl vollständig erreicht. Während weiterhin archaische Menschenarten parallel existieren (vgl. den vormals genannten $H$. naledi sowie weiterhin beispielhaft $H$. floresiensis bei Brown et al. 2004, Aiello 2010 und van den Bergh et al. 2016 sowie H. luzonensis bei Detroit et al. 2019) und in Asien die Schwesternart der Denisova-Menschen verbreitet war, so liegt der für die Sprachursprungsforschung in höherem Maße 
relevante Fokus gemeinhin auf dem paläoanthropologischen und archäologischen Vergleich der europäischen respektive westasiatischen NeandertalerPopulation mit derjenigen Linie, die hin zum modernen Menschen führt. Dies ist schlicht anhand der dort unvergleichbar umfangreichen Fundlage zu begründen. Ohne an gegebener Stelle in übermäßigem Umfang hierauf eingehen zu können, seien relevante Neuerungen dieser Spätphase dennoch wenigstens zu erwähnen und zusätzlich sollen Parallelen bzw. Unterschiede zwischen den beiden Populationen knapp skizziert werden, da sich die jüngere Forschung hier wie bereits knapp erwähnt von der lange erhaltenen Lehrmeinung distanziert.

Vor grob 250.000 Jahren begannen unsere Vorfahren, mit großem Aufwand scheinbar ästhetischen Verhaltensweisen nachzugehen - darunter fällt insbesondere die Verwendung von Farbe, zu deren Herstellung eigene Werkstätten entstanden (Brooks et al. 2018). Begehrte Materialien wie Farbe und Obsidian wurden dabei über Transportrouten von teils weit mehr als hundert Kilometern beschafft (ebd. sowie Blegen 2017). Auch der Neandertaler zeigt eine vergleichbare zeitliche Tiefe in diesen Handlungsmustern (vgl. bspw. Roebroeks et al. 2012) und produziert analog zu afrikanischen Funden seit wenigstens 130.000 Jahren Gegenstände, welche als Körperschmuck zu interpretieren sind - Ketten mit eingefärbten Muscheln, Perlenschnüre, präparierte Federn und Krallen von Greifvögeln sowie geometrische Muster auf Gegenständen (Zilhão et al. 2010, Peresani et al. 2011 und 2013, Caron et al. 2011, Morin \& Laroulandie 2012, Radovčić et al. 2015, Welker et al. 2016, Majkić et al. 2017, Majkić, d’Errco \& Stepanchuk 2018 sowie Hoffmann et al. 2018a).

Weiterhin anzuführen sind Fundstätten, an welchen Neandertaler umfangreiche Konstruktionen möglicherweise für rituelle Zwecke schufen (Jaubert et al. 2016) sowie Nachweise von Neandertaler-Begräbnissen (Valladas et al. 1987, Schwarcz et al. 1989, Pettitt 2002, Gómez-Olivencia et al. 2018 sowie zur Kontroverse mit vielseitigen Kommentaren Gargett 1989) und sowohl geometrische Muster auf Höhlenwänden (Rodríguez-Vidal et al. 2014) als auch Höhlenmalereien - und in der Tat sind die bisher ältesten bekannten Höhlenmalereien dem Neandertaler zuzuschreiben (Hoffmann et al. 2018b und 2018c). Darüber hinaus zeigte der Neandertaler entgegen früherer Annahmen das volle Spektrum der Nahrungsbeschaffung von modernen Jägern und Sammlern und ernährte sich dementsprechend umfassend von Kleinwild, Vögeln, Fisch, Seehunden, Delfinen, Nüssen, Pilzen sowie diversen Pflanzen und schreckte auch vor Bären nicht zurück (Stringer et al. 2008, El Zaatari et al. 2011, Hardy et al. 2013, Finlayson \& Finlayson 2016, Bocherens et al. 2016, Estalrrich, El Zaatari \& Rosas 
2017, Romandini et al. 2018, Morin et al. 2019 sowie Trinkhaus, Samsel \& Villotte 2019).

Nicht zuletzt stärkt sich in der jüngeren Forschung die Fundlage umfangreicher sozialer Fürsorge von Neandertalern innerhalb ihrer Gruppen zunehmend (vgl. den Überblick von Spikins et al. 2018) und es gilt weiterhin als gesichert, dass Neandertaler natürlich vorkommende Medikamente gezielt einsetzten sowie dass sie unter anderem mittels Zahnstochern ihre Mundhygiene zu stärken suchten (Hardy et al. 2012, Weyrich et al. 2017, Estalrrich, Alarcón \& Rosas 2017 sowie Hardy 2018). Als ein interessanter Seitenpunkt legt die Fundlage nahe, dass Neandertaler auf die eine oder andere Weise im Mittelmeer Seefahrten auf sich nahmen (Strasser et al. 2010, Ferentinos et al. 2012 sowie Carter et al. 2019). Für weiterführende, jedoch noch immer zusammenfassende Überblicke zum Vermögen der Neandertaler sei hiermit auf Dediu \& Levinson (2013 und 2018) sowie Roebroeks \& Soressi (2016) verwiesen. ${ }^{27}$

Vor etwa 40.000 Jahren verschwindet der Neandertaler aus Europa und wird großflächig vom modernen Menschen ersetzt (Higham et al. 2014, Villa \& Roebroeks 2014), ohne dass jedoch - entgegen der klassischen Annahme eines kognitiv überlegenen modernen Menschen - hinsichtlich dieses Zeitpunkts ein qualitativer Unterschied in Technologien, Verhalten, Kognition oder Ähnlichem zwischen den beiden Arten festgestellt werden kann (Finlayson et al. 2012, Dediu \& Levinson 2013 und 2018, Villa \& Roebroeks 2014, Roebroeks \& Soressi 2016, Hoffecker 2018). In der Tat scheint die aktuelle Fundlage $\mathrm{zu}$ implizieren, dass der Neandertaler über den Verlauf von mehreren zehntausend Jahren ein ökologisches Hindernis gegenüber einer Ausbreitung des modernen Menschen nach Europa dargestellt haben könnte. Dies wird durch die jüngere Forschung nahegelegt, nach welcher der moderne Mensch nicht erst ab vor etwa 50.000 Jahren in einer plötzlichen Revolution weltweit andere Menschenarten verdrängte, sondern tatsächlich bereits vor mehr als 100.000 Jahren über den Nahen Osten nach Indien vorstieß und bis vor spätestens 80.000 respektive 65.000 Jahren China bzw. Australien erreichte (Groucutt et al. 2015, Liu et al. 2015, Clarkson et al. 2017). Darüber hinaus zeigen Fossilfunde in Griechenland, dass der anatomisch moderne Mensch bereits vor etwa 210.000 Jahren nach Europa vorstieß, dabei jedoch wieder vom Neandertaler ersetzt bzw. zurückgedrängt wurde (vgl. Harvati et al. 2019), und genetische Befunde zeigen, dass weitere

27 Die Bedeutung dieser sowie weiterer Funde wird im Rahmen eines späteren Kapitels ausführlich diskutiert werden. An gegebener Stelle sei jedoch anzumerken, dass jene Verhaltensweisen, welche auf ein klar symbolisches Verhalten hinweisen, gemeinhin eng mit der Befähigung zu Sprache sowie dem tatsächlichen Gebrauch von Sprache korreliert werden. 
Kontaktphänomene zwischen dem Neandertaler und afrikanischen Populationen im Zeitraum von vor 460.000 und 219.000 Jahren stattgefunden haben, wobei insbesondere die mitochondriale DNA des Neandertalers beeinflusst wurde (siehe Posth et al. 2017).

\subsection{Interpretationen zur Ersetzung des Neandertalers durch den modernen Menschen}

Obwohl in den erwähnten Zeiträumen mehrere Wanderungsereignisse des H. sapiens über den Nahen und Mittleren Osten stattfanden (Cabrera et al. 2018), so konnte der moderne Mensch in Richtung des Verbreitungsgebietes des Neandertalers nicht Fuß fassen, was wie erwähnt als Hinweis darauf zu werten ist, dass der Neandertaler einen adäquaten ökologisch-demographischen Gegendruck aufrecht erhalten konnte. Die damit verbundene Schlussfolgerung einer hinsichtlich Verhalten, Kognition und Technologie vermutlich wenigstens im Groben vergleichbare Leistungsfähigkeit stützt sich mit den oben erwähnten aktuellen archäologischen und paläoanthropologischen Erkenntnissen, nach denen kein qualitatives Gefälle zwischen den Erzeugnissen und Verhaltensweisen anatomisch moderner Menschen gegenüber denjenigen des Neandertalers festgestellt werden kann (vgl. nochmals Finlayson et al. 2012, Dediu \& Levinson 2013 und 2018, Villa \& Roebroeks 2014, Roebroeks \& Soressi 2016). Für eine derartige Interpretation spricht potenziell auch der Verlauf der Verbreitung des modernen Menschen in Asien und Südostasien bis nach Australien, denn die Populationen der Denisova-Menschen, des $H$. erectus sowie weiterer, noch nicht eindeutig identifizierter Menschenarten (vgl. u.a. Nielsen et al. 2017) in Asien und Südostasien zeigten einen geringeren ökologischen Widerstand bei zugleich qualitativ und quantitativ geringerer Fundlage.

In einem knappen Exkurs soll betont werden, dass auf Basis dieser Literatur nicht von einer vollkommenen Äquivalenz zwischen dem Neandertaler und dem zeitgemäßen anatomisch modernen Menschen ausgegangen werden kann und dass noch kein großflächiger Konsens innerhalb der Forschergemeinschaft hinsichtlich der Leistungsfähigkeit des Neandertalers eingetreten ist. In diesem Sinne existieren einerseits durchaus noch immer Vertreter, darunter insbesondere Sprachursprungsforscher ohne hinreichende Einsicht in die jüngere paläoanthropologische Forschung, welche den Neandertaler dem anatomisch modernen Menschen als kognitiv klar unterlegen darzustellen suchen bzw. Zweifel an der kognitiven Leistungsfähigkeit des Neandertalers zeigen. Dagegen stellt sich die erwähnte jüngere Studienlage sowie der wachsende Konsens der paläoanthropologischen Forschungsgemeinschaft. 
Andererseits ist wie erwähnt aus der Datenlage keine vollumfängliche Äquivalenz ableitbar. Im hinreichenden Maße treffend ist die Situation wohl als „vergleichbar, aber nicht gleich“ zu beschreiben. Anscheinend gab es kein Verhalten - sei es Schmuck, Technologien, Verhaltensstrategien, Begräbnisse, Höhlenmalereien oder sonstige Dinge - , welches in $H$. sapiens und zugleich sowie quasi zeitgleich nicht im Neandertaler vorgekommen ist. Allerdings sind Nachweise moderner Verhaltensweisen anscheinend im Neandertaler zeitlich und räumlich rarer gesät, d.h. weniger systematisch in der Population integriert. Insbesondere in zeitlicher Nähe der Verdrängung des Neandertalers durch den modernen Menschen erscheint ein zunehmend großes quantitatives Ungleichgewicht, welches spätestens im Nachgang der abgeschlossenen Verdrängung des Neandertalers nicht mehr verneint werden kann.

In anderen Worten: Der moderne Mensch geht solcherlei Verhaltensweisen in gesteigertem Maße an und hinterlässt unverhältnismäßig viele Zeugnisse, während der Neandertaler zwar gleichwertige, aber weniger zahlreiche Zeugnisse hinterlässt. Die Interpretation dieser Datenlage ist noch immer höchst umstritten. Einerseits wird hier ein kognitiver und das Verhalten betreffender Trennstrich gezogen, d.h. trotz Vergleichbarkeit der Zeugnisse sei der moderne Mensch schlussendlich doch in gewisser Weise überlegen gewesen bzw. der Neandertaler schlicht nicht völlig modern gewesen. Andererseits könnte es sich hierbei um ein Datenartefakt handeln, da die Population des modernen Menschen diejenige des Neandertalers zu jenem Zeitpunkt vermutlich um grob den Faktor 10 überragte (Prüfer et al. 2014, Castellano et al. 2014, Mafessoni \& Prüfer 2017 - gegenüber einem abweichenden vorgeschlagenen Modell durch Rogers et al. 2017 - sowie nicht zuletzt Vaesen et al. 2019). Ein abschließendes Urteil ist hier noch nicht möglich, scheint jedoch zunehmend in greifbare Nähe zu rücken.

Wie soeben erwähnt zeigen jüngere Studien, dass die Gesamtpopulation des Neandertalers bis zur Ausbreitung des modernen Menschen nach Europa um eine Größenordnung geringer ausfiel als diejenige des $H$. sapiens. In diesem Sinne war die Population der Neandertaler vergleichsweise anfällig sowohl gegenüber ökologischen Fluktuationen als auch gegenüber einem zunehmenden demographischen Ungleichgewicht zugunsten des modernen Menschen (vgl. nochmals die soeben Genannten). Diese Umstände allein könnten als Erklärung für ein Zusammenbrechen des beschriebenen ökologischen Gleichgewichts vor etwa 40.000 Jahren ausreichen (vgl. Vorgenannte sowie Kolodny \& Feldman 2017, Goldfield, Booton \& Marston 2018 und Degioanni et al. 2019), wurden jedoch anscheinend auch klimatisch bedingt - innerhalb einer für Neandertaler letztendlich äußerst ungünstigen teilweisen Entvölkerung Europas, 
in deren Wiederbevölkerung Faktoren wie die soeben genannten dem modernen Menschen schlicht die Oberhand zuspielten (vgl. bspw. Alcaraz-Castano et al. 2017, Wolf et al. 2018, Melchionna et al. 2018 sowie Staubwasser et al. 2018).

Im weiteren zeitlichen Verlauf ersetzte der moderne Mensch den Neandertaler großflächig - jedoch im Rahmen einer umfangreichen genetischen Vermischung der Populationen (für jüngere Studien, die diese Feststellung stützen McCoy, Wakefield \& Akey 2017 und Lipson \& Reich 2017; für Studien zur positiven Selektion auf Neandertaler- und Denisova-Gene Dannemann \& Kelso 2017, Racimo, Marnetto \& Huerta-Sánchez 2017, Gittelman et al. 2017 sowie Enard \& Petrov 2018; für umfangreiche Reviews der einschlägigen Forschungslage Nielsen et al. 2017 und Dannemann \& Racimo 2018). Zusätzlich geschah eine vergleichbare genetische Durchmischung auch in kontinentalasiatischen und südostasiatischen Regionen mit den Populationen der Denisova-Menschen sowie weiteren archaischen Populationen (vgl. nochmals Nielsen et al. 2017 sowie Dannemann \& Racimo 2018), wobei auch zwischen westasiatischen Neandertalern und Denisova-Menschen Kontakt bestand (Slon et al. 2018).

Im Nachgang dieses weltweiten Ablösens ,archaischer“ menschlicher Populationen durch den modernen Menschen findet schließlich die vorliegende Skizze der Menschwerdung ihr Ende, da innerhalb der Sprachursprungsforschung eine Befähigung sowie Fertigkeit zu voll ausgebildeter moderner Sprache bei $H$. sapiens zu jenem Zeitpunkt weitestgehend unumstritten ist. Während eine genauere Datierung des Sprachursprungs sich jenseits dieser Feststellung als äußerst schwierig zeigt, so wird an späterer Stelle eine entsprechende Diskussion wenigstens zu versuchen sein. Nachfolgend steht jedoch zunächst die letzte der drei fachlichen Einführungen im Vordergrund und im Anschluss hieran sollen die Forschungsfragen, Hypothesen und Methoden der vorliegenden Arbeit angemessen expliziert werden. 